Plasmon resonant cavities in vertical nanowire arrays

M. BOra, T. BOnd, E.Behymer, A. Chang

March 5, 2010

NanoLetters 
This document was prepared as an account of work sponsored by an agency of the United States government. Neither the United States government nor Lawrence Livermore National Security, LLC, nor any of their employees makes any warranty, expressed or implied, or assumes any legal liability or responsibility for the accuracy, completeness, or usefulness of any information, apparatus, product, or process disclosed, or represents that its use would not infringe privately owned rights. Reference herein to any specific commercial product, process, or service by trade name, trademark, manufacturer, or otherwise does not necessarily constitute or imply its endorsement, recommendation, or favoring by the United States government or Lawrence Livermore National Security, LLC. The views and opinions of authors expressed herein do not necessarily state or reflect those of the United States government or Lawrence Livermore National Security, LLC, and shall not be used for advertising or product endorsement purposes. 


\title{
Plasmon resonant cavities in vertical nanowire arrays
}

Mihail Bora*, Benjamin J. Fasenfest, Elaine M. Behymer, Allan S-P Chang, Hoang T. Nguyen, Jerry A.

Britten, Cindy C. Larson, Tiziana C. Bond*

${ }^{1}$ Lawrence Livermore National Laboratory, Livermore CA, USA

Corresponding authors: bora1@1lnl.gov, bond7@1lnl.gov

\begin{abstract}
We investigate tunable plasmon resonant cavity arrays in paired parallel nanowire waveguides. Resonances are observed when the waveguide length is an odd multiple of quarter plasmon wavelengths, consistent with boundary conditions of node and antinode at the ends. Two nanowire waveguides satisfy the dispersion relation of a planar metal-dielectric-metal waveguide of equivalent width equal to the square field average weighted gap. Confinement factors over $10^{3}$ are possible due to plasmon focusing in the inter-wire space.
\end{abstract}


Surface plasmons, charge density waves propagating along metal-dielectric interfaces are applied in bio-molecular detection, ${ }^{1-3}$ sub-wavelength optics $^{6-8}$ and photovoltaic technology. ${ }^{9-11}$ Different geometries have been employed to study plasmonic structures. Horizontal layer approach provides excellent thickness control, but results in fewer plasmonic nanostructures per unit area. ${ }^{12}$ The vertical structures provide good coverage, but require complex fabrication and are less amenable to large area substrates. ${ }^{13}$ Here we propose fabrication of tunable plasmon resonant cavities in vertical wire arrays that combines the benefits of both approaches providing high density and good control over the cavity size over a large area (4 inch wafer) uniform plasmonic substrate. Tuning of the plasmon resonance in the $500-800 \mathrm{~nm}$ range is demonstrated by controlling the geometrical dimensions of the cavity. Future applications for the nano-cavities are envisioned in high sensitivity Raman spectroscopy ${ }^{4-5}$ that requires high local electromagnetic fields and alignment between the plasmon resonance and excited and scattered light. The tunable nanocavity is of particular relevance for fabrication of plasmonic lasers which use surface plasmons instead of light to pump the lasing medium. Since the device structure relies on vertical free standing nano-wires the plasmonic cavity region can be filled with any material of choice. In addition to the high confinement factors shown, the cavity plasmon resonance can be adjusted for maximum overlap with the absorbance of the active material. 

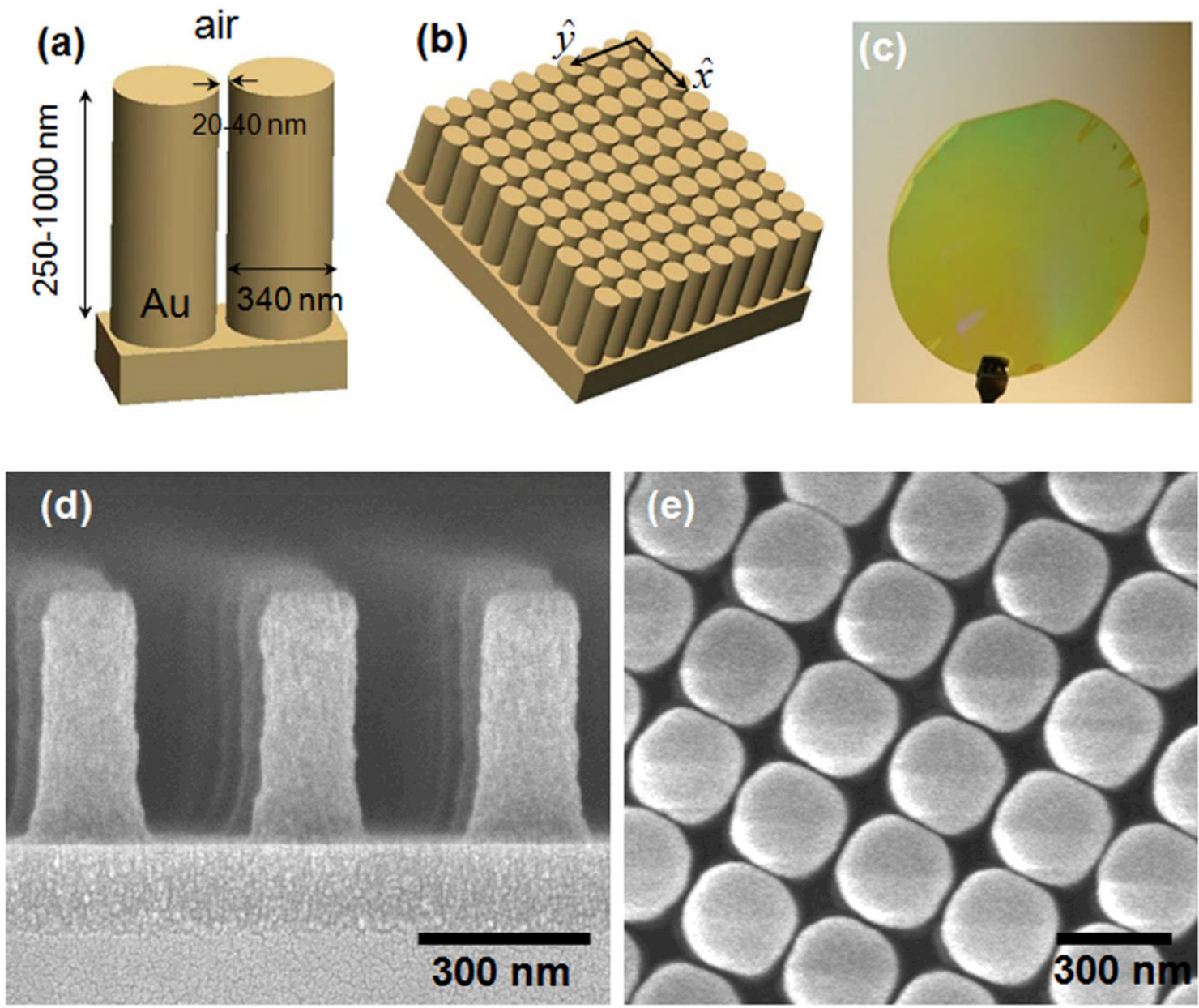

Figure 1. Vertical nanowire array substrate. (a) Single plasmon resonant cavity based on a twonanowire waveguide. The separation medium is air of dielectric permittivity 1 . Nanowire thickness is approximately $340 \mathrm{~nm}$, and the height ranges from 250 to $1000 \mathrm{~nm}$. The wavevector of the incident light is oriented along the waveguide in the transverse magnetic mode (b) Rectangular array of cavities on a planar substrate. Arrows indicate the lattice vectors of the array. (c) Large area fabrication of the plasmon nanocavities on a four-inch diameter substrate. (d) Side view of photoresist nanowires fabricated by laser interference lithography. (e) Top view of metallic nanowire cavity array coated with gold. The wire center-to-center distance is $360 \mathrm{~nm}$ and the edge to edge gap is $35 \mathrm{~nm}$.

The resonant cavity relies on paired nanowire waveguides that supports propagation of gap plasmon modes $^{14-15}$ (Figure 1a) when the separation is less than $50 \mathrm{~nm}$. The cavity is delimited by a reflective metallic mirror at one side and an open end on the other for a more effective incident light-plasmon coupling. ${ }^{16}$ Resonances occur when the cavity length is an odd multiple of quarter plasmon wavelengths 
due to boundary conditions of node at the mirror and anti-node at the open end. A two dimensional rectangular array of vertical resonant cavities is fabricated on a planar substrate (Figure 1b-e). Each nanowire is surrounded by four closest neighbors corresponding to two resonant cavities per nanowire, for a density of $3.85 \cdot 10^{8}$ cavities $/ \mathrm{cm}^{2}$. The vertical wire geometry enables large area fabrication (Figure 1c) and far field optical characterization. No inter-coupling between cavities is expected in these structures, since the size of the nanowires is an order of magnitude larger than the penetration depth of the electric field in the metal.

Photoresist wire arrays are patterned on a fused silica substrate using laser interference lithography under conditions described previously. ${ }^{17}$ The pattern was the transferred on the silica substrate by reactive ion etching. The silica nanowires are coated with alumina by atomic layer deposition so that inter-wires gap is approximately $75 \mathrm{~nm}$. Since the alumina deposition is self limiting, the thickness can be increased in conformal single molecule layer steps. Finally, a 20-40 nm thick gold coating was deposited by sputtering until the edge to edge distance between adjacent wires approaches 20-40 $\mathrm{nm}$.
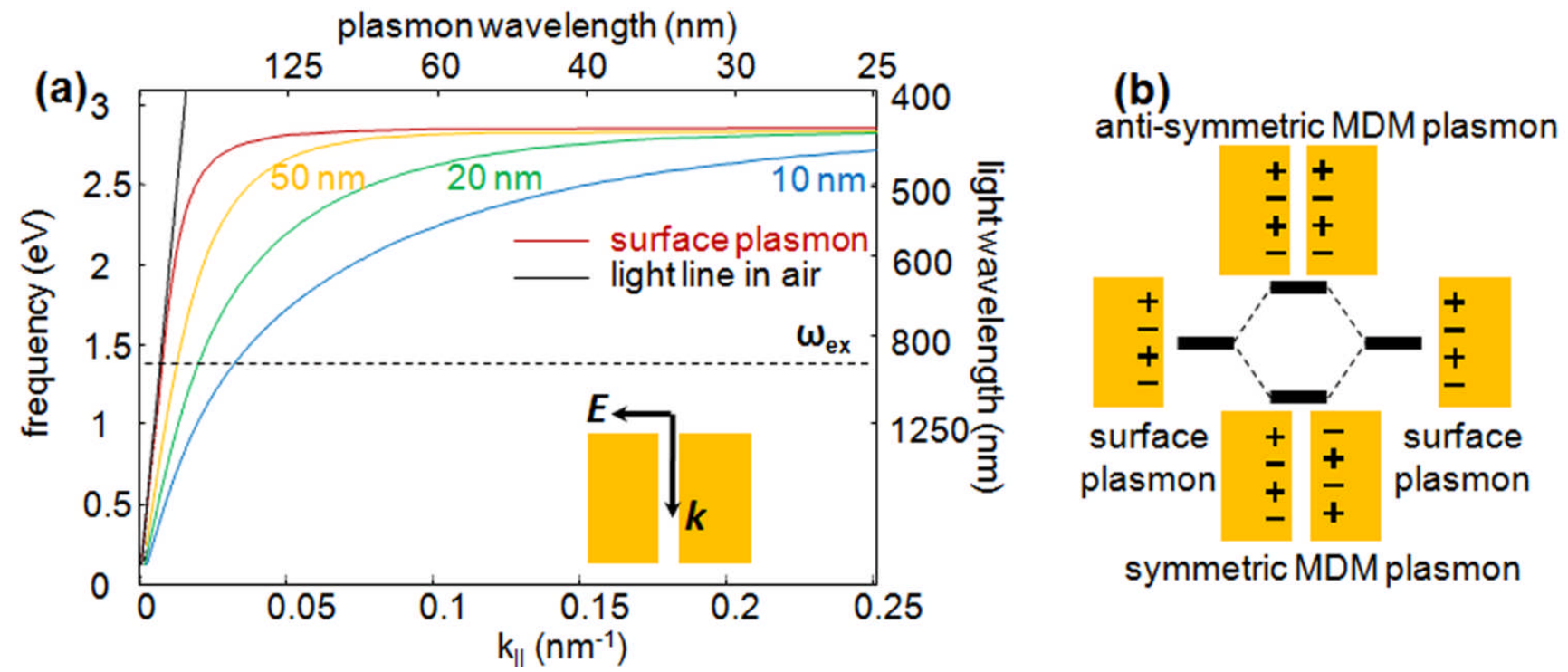

Figure 2. Planar MDM waveguide. (a) Dispersion relations for the gold-air-gold (MDM) symmetric plasmon for different air thicknesses. The numbers near the curves indicate the width of the dielectric (air). For incident light along the waveguide of frequency $\omega_{\mathrm{ex}}$, plasmon modes of the same frequency and wavevector given by dispersion curve are excited. Inset: schematic representing the MDM waveguide. The plasmon modes are excited by light propagating with wavevector $k$, along the planar 
waveguide with the electric field vector $E$, perpendicular to the metal surface. (b) Qualitative behavior of the MDM plasmon based on the hybridization model. When two elementary thin film plasmons are brought in close proximity two new states are formed: a symmetric (low energy) and antisymmetric (high energy state).

The two nanowire plasmon waveguide properties can be explained using an underlying planar metaldielectric-metal (MDM) waveguide that is composed of two semi-infinite metal volumes separated by a dielectric of finite thickness (Figure 2a, inset). The MDM waveguide is sufficiently simple to allow an analytical calculation of the dispersion curve based on the geometry and materials dielectric properties. The two nanowire waveguide is approximated as an MDM waveguide of effective width $\mathrm{w}_{\mathrm{eff}}$ that is calculated using the appropriate gap weighting metric. For example, for the optical extinction coefficient, the effective width is equal to the gap weighted with the square average of the field strength. The technique of decomposing the two nanowire waveguide into a continuum of elementary planar waveguides can be extended to other geometries providing a straightforward way to calculate the dispersion relation of arbitrary shape waveguides (e.g. wedges, grooves).

Dispersion relations for MDM plasmons are determined by solving for propagating modes along the waveguide under continuity conditions at metal dielectric boundaries while satisfying Maxwell's equations. ${ }^{18-19}$ The MDM solutions degenerate into symmetric and anti-symmetric modes, defined by the parity of the electric field in the direction perpendicular to the dielectric sheet, with respect to the mid-plane. Single interface surface plasmon modes are recovered when the separation is increased beyond the evanescent decay length.

The plasmon degeneracy can be understood in the plasmon hybridization model ${ }^{20}$ (Figure $2 \mathrm{~b}$ ) in which the MDM plasmon is decomposed into two interacting single metal interface plasmons. The low energy or "bonding" state corresponds to the symmetric mode and the high energy or "anti-bonding" state to the anti-symmetric mode. 
The coupling efficiency between light and MDM plasmons is proportional with the spatial and temporal overlap of the modes ${ }^{21}$ :

$$
\eta=\frac{\left\langle H_{\text {plasmon }} \mid H_{\text {photon }}\right\rangle^{2}}{\left|H_{\text {plasmon }}\right|^{2} \cdot\left|H_{\text {photon }}\right|^{2}}
$$

where $\boldsymbol{\eta}$ is the coupling efficiency and $H_{\text {plasmon }}$ and $H_{\text {photon }}$ are the field profiles for the plasmon and electromagnetic plane wave excitation modes. The electric field of the incident light is constant across the gap (symmetric) if the dielectric width is much smaller than the light wavelength; hence, the coupling efficiency is non-zero only for the symmetric mode.

The dispersion relation for the symmetric mode is given by the equation: ${ }^{18}$

$$
\tanh \left(i k_{\perp}^{d} \frac{w}{2}\right)=-\frac{\varepsilon_{d} k_{\perp}^{m}}{\varepsilon_{m} k_{\perp}^{d}}
$$

where $k_{\perp}^{d}$ and $k_{\perp}^{m}$ are the perpendicular components of the plasmon wavevector in the dielectric and metal, $\varepsilon_{d}$ and $\varepsilon_{m}$ are the relative permittivities for the dielectric and metal, respectively, and $w$ is the width of the dielectric sheet. In Figure 2a, the dispersion relations for gold-air-gold waveguides of different air thicknesses are plotted. The dielectric function of gold is based on a Drude model fit for the published optical constants of gold. ${ }^{22}$
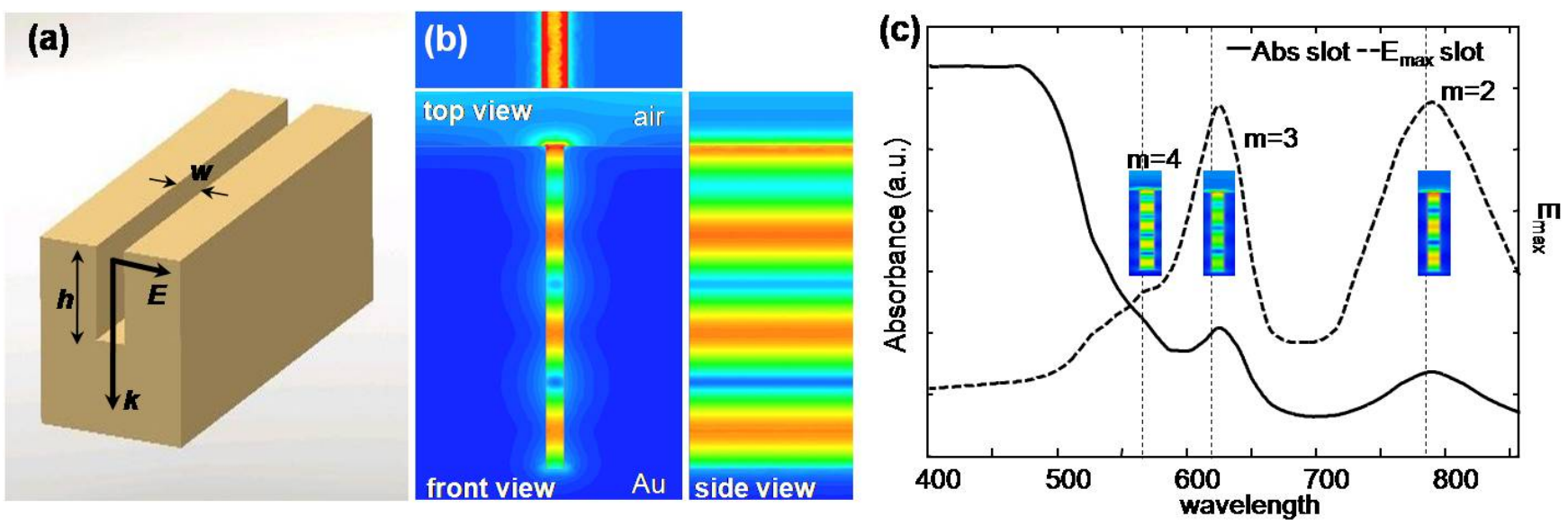

Figure 3. (a) Three dimensional model of the MDM infinite slot waveguide. The plasmon modes are excited by transverse magnetic polarized light of normal incidence to the metallic plane. Dark arrows indicate the orientation of the wavevector and electric field of the excitation light. (b) Numerical 
simulation of the electric field amplitude in the slot from the front, side and top. (c) Maximum electric field amplitude in the cavity normalized to the incident light field and optical absorbance of the slot as a function of wavelength. Resonances of order 2 and 3 and 4 are observed at 800, 650 and $565 \mathrm{~nm}$ in both absorbance and electric field plots. Bellow $600 \mathrm{~nm}$ the material absorbance of gold dominates the optical properties of the resonator. Dashed vertical lines indicate the position of the resonance calculated from Equation 2. The small discrepancy with the numerical simulation is attributed to a phase change at the ends of the cavity. The cavity orders are illustrated by insets representing the electric field amplitude for each resonance.

Two different cavity types are analyzed. The first, the MDM slot waveguide, is a rectangular groove of height $\mathrm{h}$ and width $\mathrm{w}$ and infinite length indented in a semi-infinite metallic volume (Figure $3 \mathrm{a}$ ). This ideal structure allows direct comparison with the exact analytical dispersion relation calculated above for the MDM waveguide. The second case considered is a vertical wire nanocavity, composed of two metallic cylinders separated by a small distance (Figure 4a). The plasmonic features of vertical wire nanocavity are similar to those of the MDM slot waveguide, both showing resonances when the length of the cavity is an odd multiple on quarter plasmon wavelengths. Furthermore, the two nanowire waveguide satisfies the dispersion relation a modified planar MDM waveguide.

The two cavities are modeled as a waveguide of finite length, delimited by a metallic interface at the bottom and dielectric on top. Plasmon MDM type modes are excited by light normally incident on the open end side of the cavity. The photon field overlaps with the plasmon cavity over a thin region with a thickness comparable to the skin depth of gold. The free electrons in the metal oscillate with the excitation light frequency $\omega_{\mathrm{ex}}$ and plasmon waves with wavevector given by the dispersion relation calculated in Equation 2 propagate along the waveguide. On the closed metallic end, a reflection with a $\pi$ phase shift occurs and a plasmonic standing wave forms along the waveguide.

The structure resonant features are derived from the boundary conditions at the metal mirror (antinode) and open dielectric side (node): 


$$
k h=\pi\left(m+\frac{1}{2}\right)
$$

where $k$ is the plasmon wavevector along the inter-wire region, $h$ is the wire height, and $m=0,1,2 \ldots$ is the resonance order. The plasmon states for which the wavevector satisfies the resonance condition in Equation 3 couple more strongly with the incident light and have a higher extinction coefficient, resulting in minima in the reflection spectrum.

Numerical simulations were carried for MDM slot waveguides of height $500 \mathrm{~nm}$ and width $20 \mathrm{~nm}$ under excitation light normally incident on the substrate surface in the transverse magnetic mode. Resonant modes are observed in the plots of the electric field amplitude when standing plasmonic waves are formed, (Figure 3c). Peaks are observed for resonances of order $m=2(785 \mathrm{~nm}), \mathrm{m}=3(620 \mathrm{~nm})$ and $\mathrm{m}=4(565 \mathrm{~nm})$. The wavelength dependence of the substrate absorbance, calculated as the heat dissipation in the metallic material, and the electric field amplitude show peaks at the same location. This finding allows correlation of the near-field properties of the plasmonic cavity using far field characterization techniques such as reflectance and transmission.
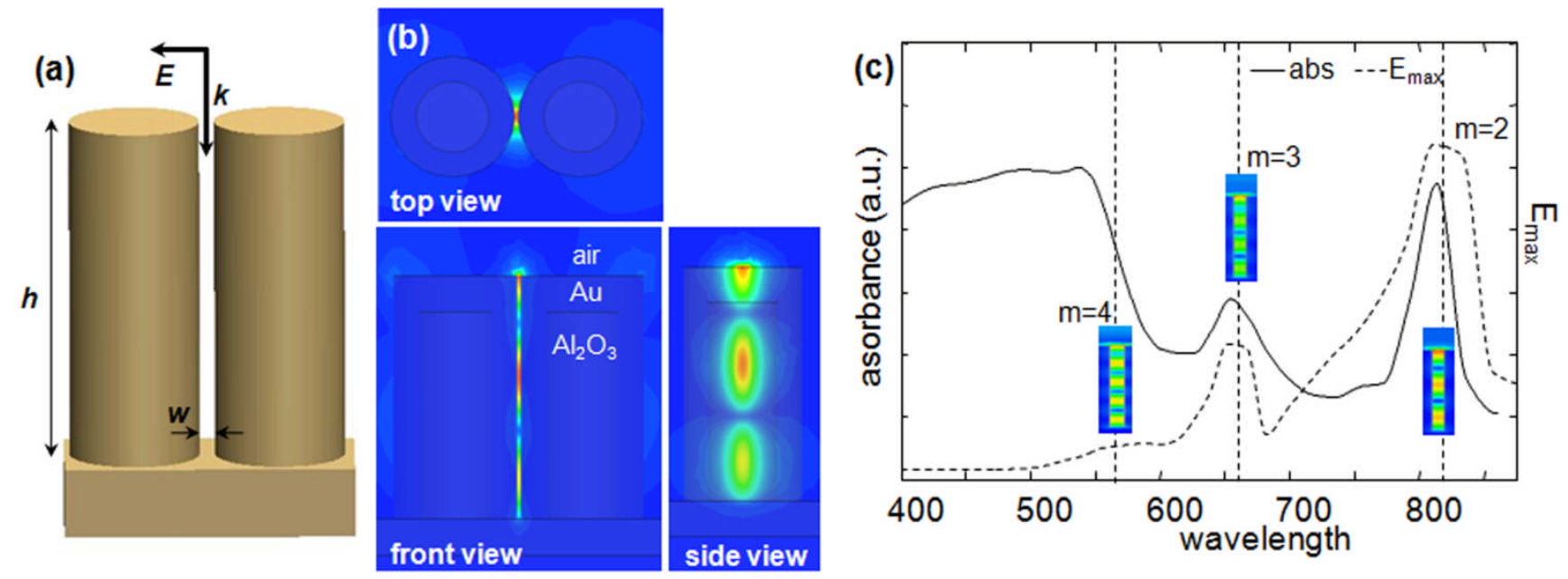

Figure 4. (a) Two-nanowire plasmon resonant cavity. The incident light field is oriented normal to the side surface of the wires (transverse magnetic mode). (b) Numerical simulations of the electric field as viewed from the front, top and side directions. (c) Resonances of order 2 and 3 and 4 are observed at 800,650 and $565 \mathrm{~nm}$. 
A three-dimensional simulation of the two nanowire cavity was compared with the MDM slot model (Figure 4). Similar characteristics were observed for the electric field profile, and absorbance spectrum. As in the previous simulations the plasmon modes are excited by transverse magnetic normal incident light. Resonances are observed when the height of the wire is an odd multiple of quarter plasmon wavelengths. The plasmon modes are strongly confined to the inter-wire space where the amplitude of the electric field is two orders of magnitude larger than in the rest of the structure.

(a)

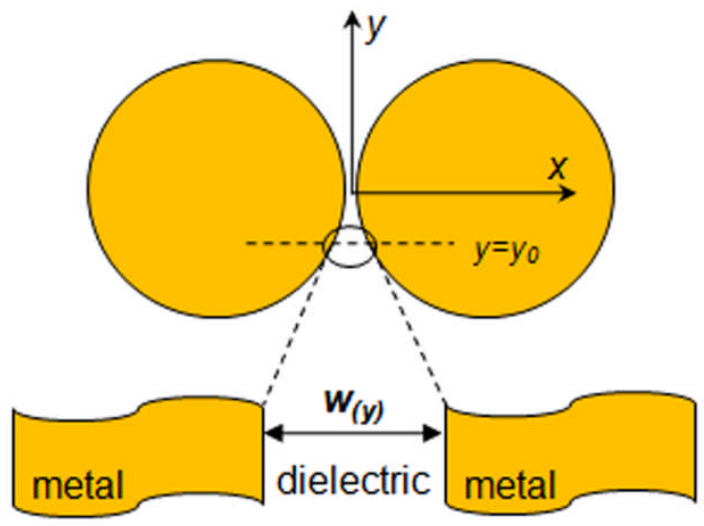

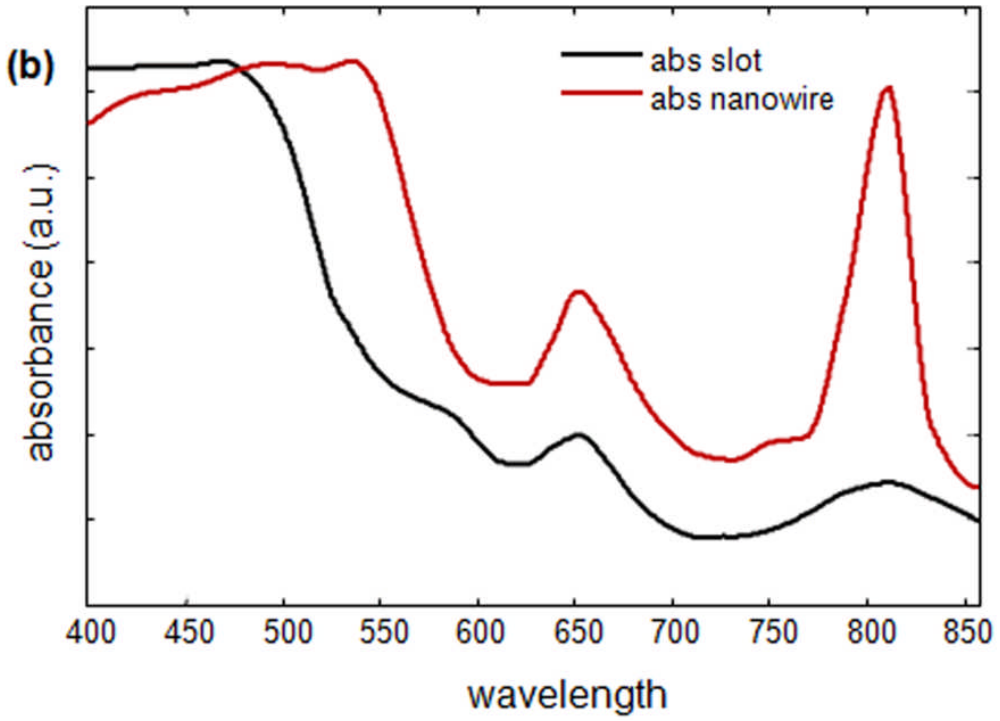

Figure 5. Equivalence between the two nanowire and the MDM slot waveguide. (a) Top view of the two nanowires. The wire waveguide can be approximated for each point along the circumference with a planar MDM waveguide. An effective width is calculated by averaging the variable width over the square of the filed in the gap. (b) Comparison of absorbance spectra and electric field maximum amplitude between nano-wires (red) and slot (black) cavities having the same effective width.

The MDM slot model predicts the optical properties of the two nanowire cavity when the equivalent spacing is taken into account (Figure 5). The effective distance between the wires can be calculated using coupling mode theory (Equation 1). Assuming that both photon and plasmon magnetic fields are normalized to unity and that the photon field is nearly constant across the gap, the coupling efficiency at each point along the $\mathrm{y}$ axis, Figure $5 \mathrm{a}$, is given by: 


$$
\eta_{\left(y_{0}\right)} \propto\left(\int_{y=y_{0}} H_{\text {plasmon }}^{y} d x d z\right)^{2}
$$

where $H_{\text {plasmon }}^{y}$ is the $y$ component of the plasmon magnetic field ( $x$ and $z$ components are null). The coupling efficiency between photons and plasmons is therefore proportional to the square of the field average. Based on coupling mode theory argument presented above, we can calculate the effective separation of two adjacent cylinders as the average width weighted by the metric described earlier. This key finding allows derivation of the dispersion relation of complex plasmonic waveguides using a much simpler planar waveguide. Although outside the inter-wire region the cylindrical geometry deviates from the parallel interface model, $90 \%$ of the total electromagnetic field energy is concentrated in a circular sector with a \pm 12 degrees opening. For the dimensions fabricated array (wire center-to-center distance of $360 \mathrm{~nm})$, the effective width is approximately two times the edge to edge wire distance (15$35 \mathrm{~nm}$ ). In Figure 5b we compare the extinction spectrum for the slot cavity in Figure $3 \mathrm{c}$ with that of the nanowire cavity in Figure 4c. Wire cavities that resonate at the same frequency as the slot cavities show a higher maximum electric field and extinction coefficient. This can be explained by an increase in the surface charge density which is inversely proportional to the radius of curvature.

Although the plasmon cavity modes are excited only by light polarized in the transverse magnetic mode (i.e. the electric field vector is oriented along the wire center to center direction) the wire array optical properties are independent of polarization. The substrate contains two sets of plasmonic cavities aligned along the two perpendicular lattice vectors, Figure 1b. The TM mode of the incident light as referenced to the first subset $(\hat{x})$, corresponds to the TE mode for the second one $(\hat{y})$, and orthogonal decomposition of the incident electric field with respect to the two axes explains the polarization invariance.

It is useful to compare the mode profile of the MDM slot in Figure 3b with the same order mode for the wire cavity in Figure 4b. Although the front, top and side views of the MDM slot are redundant, they are included for a more clear comparison with the wire cavity mode. The two-nanowire plasmons are 
confined in both lateral dimensions; along the direction normal to the wire surface the confinement is determined by the charge screening of the electric field in the metal layer. The confinement in the second lateral direction results from the formation of a plasmon energy trap. Opposite polarity surface charges of the symmetric mode minimize the dipole interaction energy when the distance is shortest. Since the effective MDM waveguide separation is increasing rapidly away from the inter-wire region, a strong energy gradient will focus the plasmons in the inter-wire space. This self-confinement feature makes the two wire plasmonic waveguides ideal candidates for high density plasmonic interconnects since the packaging density is increased significantly. Three dimensional sub-wavelength confinement becomes possible if the cavity length is smaller than the wavelength of the excitation light. For the 250 $\mathrm{nm}$ tall cavities we estimate the confinement factor to be approximately 1800 .

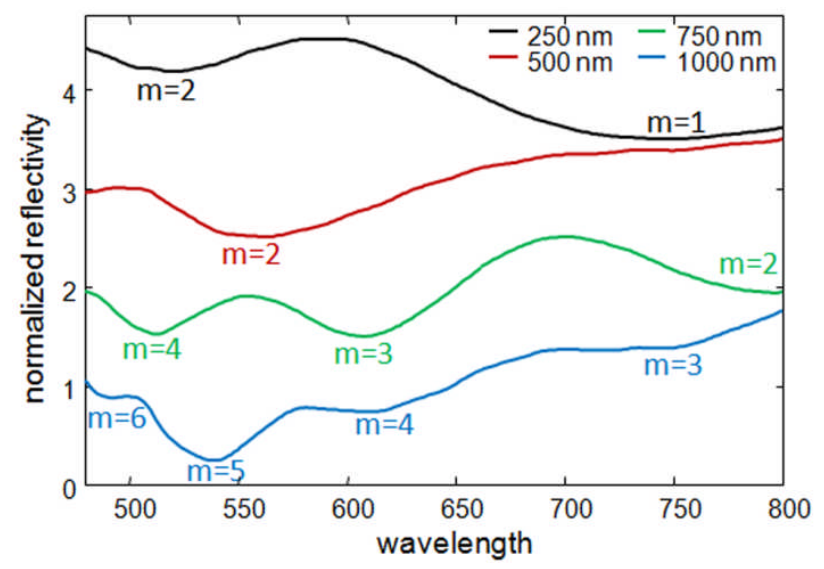

Figure 6. Normalized reflectivity plots for wire array of height 250, 500, 750 and $1000 \mathrm{~nm}$. Incident light is non-polarized and perpendicular to the substrate. The reflectivity minima correspond to nanocavity excitation of plasmon resonances labeled by their order $\mathrm{m}$.

The nanowire cavity array substrates were optically characterized by measuring the wavelength dependent normal incidence reflectivity with a NanoSpec 3000 tabletop film analysis system (Nanometrics Inc., Milpitas, CA). Reflectivity plots were normalized to a $100 \mathrm{~nm}$ thick gold film to account for material specific absorbance (Figure 6). Excitation of plasmons within the cavity increases the extinction coefficient of the substrate and resonances are observed as minima in the reflectivity data. 
The cavity order was identified using numerical simulations and the analytical expression of the dispersion curve.

The plasmon resonance condition can be changed by modifying either the wire height or separation. The first will alter the cavity length, while the second will change the plasmon propagating wavevector according to the dispersion relation. For a vertical wire cavity of given height and separation, resonances of monotonically decreasing order are observed with increasing excitation wavelength, the highest order resonances being located towards the blue side of the spectrum.
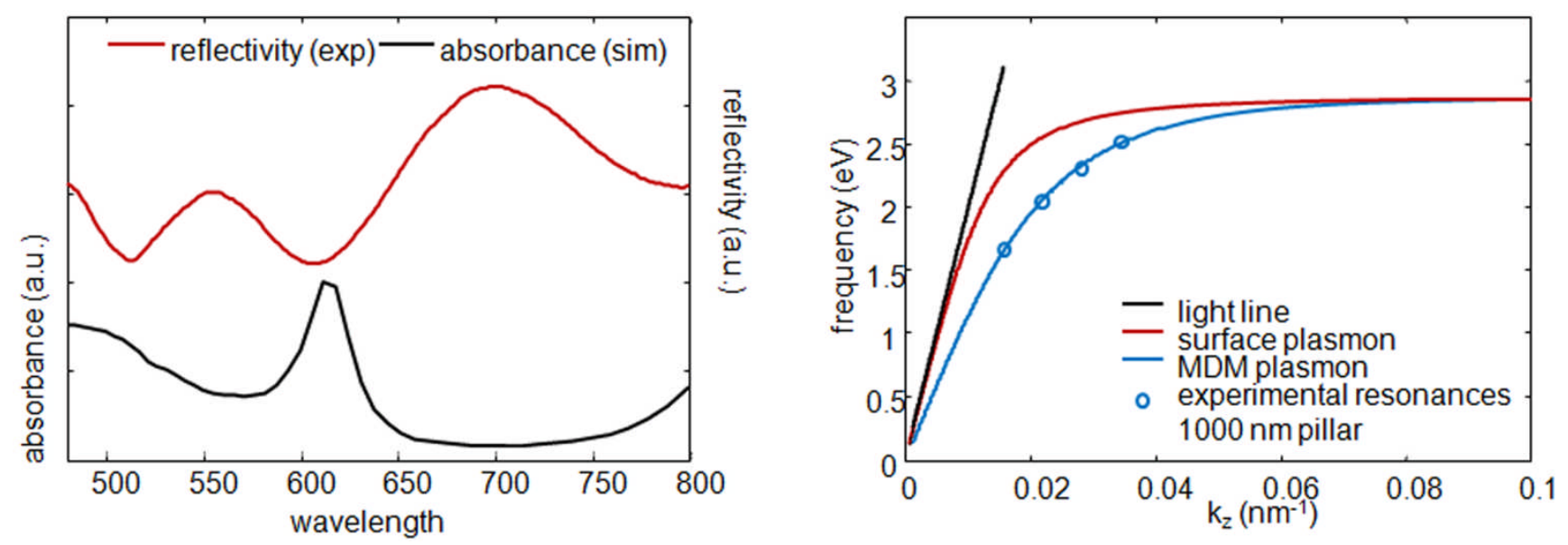

Figure 7. (a) Measured reflectivity and simulated absorbance in the nanowire array. The minima of the experimental reflectivity align with the simulated absorbance maxima. (b) Analytical and experimental dispersion relations. Solid blue curve: analytical calculation based on MDM infinite waveguide using an effective gap separation. Blue circles: experimental points calculated from the $1000 \mathrm{~nm}$ tall wire reflectivity measurements.

Numerical simulations of the far and near field patterns show good agreement with the experimental data. Measured reflectivity was compared with numerical simulations of the absorbance (Figure 7a). Resonances are observed as minima in the reflectivity plots and as maxima in the absorbance simulations. Typically experimental data has a lower resonance quality factors then numerical simulations, probably because of additional roughness effects. Below $550 \mathrm{~nm}$ there is a discrepancy 
between simulation and experiment. We speculate that in this spectral range the optical properties of the 20-40 nm gold coating differ from the bulk values used in the modeling.

The plasmon wavelength can be calculated from Equation 3, knowing the cavity length and cavity order. The resonances measured in the experimental reflectivity are compared with numerical simulations to determine the resonance order and to calculate the plasmon wavevector. Further, the wavevector and frequency points are plotted along the dispersion curve for the MDM waveguide (Figure 7b) from the analytical expression in Equation 2, using a separation value that best fitted the data. The good agreement between the analytical calculation of dispersion relation, numerical simulation of electric field and absorbance, and experimental data reflectivity show that the twonanowire plasmon nanocavity can be approximated using a variable width MDM waveguide. The nanowire geometry allows for a stronger confinement resulting in higher fields and quality factors.

In conclusion, we demonstrate plasmonic resonant cavities that are supported along a two nanowire waveguide. Three dimensional subwavelength confinement is realized by adjusting the longitudinal length of the cavity for lower order resonance, and by focusing the plasmon modes between two adjacent wires in an energy gradient. Confinement factors over of $10^{3}$ are realized for the $1^{\text {st }}$ order resonance of the $250 \mathrm{~nm}$ tall cavity considered, however this factor can be increased to considerably larger values using smaller height and diameter wires and shorter wavelength excitation light. We have also shown that the optical properties of complex plasmonic structures (e.g. two-nanowire waveguides) can be derived from elementary structures (MDM planar waveguide) by using a squared field average weight for the effective geometrical dimensions. The high electric field in the cavity and the tunability of the plasmon resonance with height and separation make cavity based substrates promising candidates for surface enhanced Raman spectroscopy and plasmonic lasers.

Aknowledgement. This work performed under the auspices of the U.S. Department of Energy by Lawrence Livermore National Laboratory under Contract DE-AC52-07NA27344. LLNL-ABS-420921 


\section{References}

(1) Hirsch, L. R.; Jackson, J. B.; Lee, A.; Halas, N. J.; West, J. Analytical Chemistry 2003, $75,2377$.

(2) Rich, R. L.; Myszka, D. G. Current Opinion in Biotechnology 2000, 11, 54.

(3) Bora, M.; Celebi, K.; Zuniga, J.; Watson, C.; Milaninia, K. M.; Baldo, M. A. Optics Express 2009, 17, 329.

(4) Haynes, C. L.; Van Duyne, R. P. Journal of Physical Chemistry B 2003, 107, 7426.

(5) Michaels, A. M.; Nirmal, M.; Brus, L. E. Journal of the American Chemical Society 1999, 121, 9932.

(6) Barnes, W. L.; Dereux, A.; Ebbesen, T. W. Nature 2003, 424, 824.

(7) Ghaemi, H. F.; Thio, T.; Grupp, D. E.; Ebbesen, T. W.; Lezec, H. J. Physical Review B 1998, 58, 6779 .

(8) Lezec, H. J.; Degiron, A.; Devaux, E.; Linke, R. A.; Martin-Moreno, L.; Garcia-Vidal, F. J.; Ebbesen, T. W. Science 2002, 297, 820.

(9) Morfa, A. J.; Rowlen, K. L.; Reilly, T. H.; Romero, M. J.; van de Lagemaat, J. Applied Physics Letters 2008, 92.

(10) Tvingstedt, K.; Persson, N. K.; Inganas, O.; Rahachou, A.; Zozoulenko, I. V. Applied Physics Letters 2007, 91. 
(11) Westphalen, M.; Kreibig, U.; Rostalski, J.; Luth, H.; Meissner, D. Solar Energy Materials and Solar Cells 2000, 61, 97.

(12) Miyazaki, H. T.; Kurokawa, Y. Applied Physics Letters 2006, 89.

(13) Haynes, C. L.; Van Duyne, R. P. Journal of Physical Chemistry B 2001, 105, 5599.

(14) Manjavacas, A.; de Abajo, F. J. G. Nano Letters 2009, 9, 1285.

(15) Manjavacas, A.; de Abajo, F. J. G. Optics Express 2009, 17, 19401.

(16) Stegeman, G. I.; Wallis, R. F.; Maradudin, A. A. Optics Letters 1983, 8, 386.

(17) Fernandez, A.; Nguyen, H. T.; Britten, J. A.; Boyd, R. D.; Perry, M. D.; Kania, D. R.; Hawryluk, A. M. Journal of Vacuum Science \& Technology B 1997, 15, 729.

(18) Dionne, J. A.; Sweatlock, L. A.; Atwater, H. A.; Polman, A. Physical Review B 2006, 73.

(19) Lezec, H. J.; Dionne, J. A.; Atwater, H. A. Science 2007, 316, 430.

(20) Prodan, E.; Radloff, C.; Halas, N. J.; Nordlander, P. Science 2003, 302, 419.

(21) Sun, Z. J.; Zeng, D. Y. Journal of the Optical Society of America B-Optical Physics 2007, 24, 2883.

(22) Johnson, P. B.; Christy, R. W. Physical Review B 1972, 6, 4370. 